\title{
ACCESS: AUSTRALIA'S CONTRIBUTION TO THE ISERVO INSTITUTE'S DEVELOPMENT
}

\begin{abstract}
The new Australian Computational Earth Systems Simulator research facility provides a virtual laboratory for studying the solid earth and its complex system behavior. The facility's capabilities complement those developed by overseas groups, thereby creating the infrastructure for an international computational solid earth research virtual observatory.
\end{abstract}

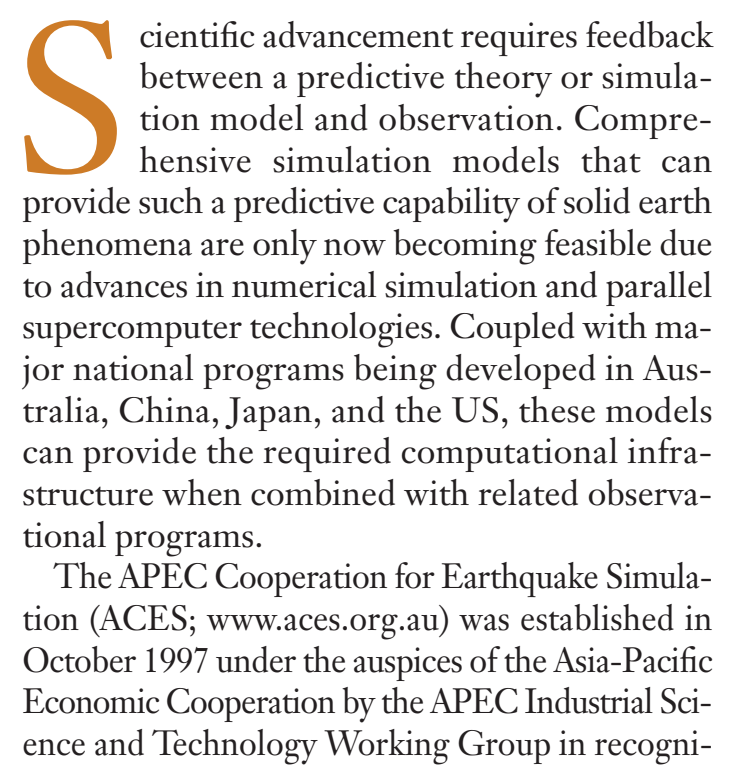

$1521-9615 / 05 / \$ 20.00$ ○ 2005 IEEE

Copublished by the IEEE CS and the AIP

Peter Mora, Hans MüHlhaus, Lutz Gross,

Huilin Xing, AND Dion WeAtherley

University of Queensland, Australia

STEFFEn ABE AND SHANE LATHAM

Australian Computational Earth Systems Simulator

LOUIS MORESI

Monash University tion of this new opportunity and the complementary strengths of the different national research programs. ACES aims to develop simulation models for the complete physics of earthquakes, foster collaboration between the complementary national programs, and foster development of a research infrastructure (supercomputers, software, and numerical methods for earth simulation). ACES brings together observational, theoretical, and computational seismologists; computational scientists; physicists; geologists; computer scientists; and laboratory researchers to work toward this common goal. ${ }^{1,2}$

As a follow up to this international cooperation, ACES participants have agreed to establish the international Solid Earth Research Virtual Observatory (iSERVO), a frontier institute for solid earth systems research (see the "iSERVO: International Institute" sidebar for more details). iSERVO will extensively use Web and Grid technologies to let both researchers affiliated with the institute and external users effectively collaborate and run the simulation models and software the institute develops. Australia's contribution involves a new national research facility-the Australian Computational Earth Systems Simulator (ACcESS). The new facility will consist of a high-level computational framework, parallel software, and supercomputer hardware for solid earth simulation. The ACcESS simulator facility includes capabilities to model rocks and granular systems at the particle scale, the 


\section{iSERVO: International Institute}

ince the commencement of ACES, Japan has established the Earth Simulator-the world's fastest supercomputer at the time of its installation-for simulating Earth processes and has established a new Center of Excellence for predicting the multiscale earth system's evolution and variation. Australia's Australian Computational Earth Systems Simulator (ACCESS) was funded with the goal of developing numerical models, parallel software, and supercomputer hardware to enable large-scale simulation of solid earth processes from the microscopic to the global scale. In the US, NASA's Jet Propulsion Laboratory, in collaboration with the GEM Group and national science centers, is developing a computational infrastructure to model the physics of the California fault system. In China, national programs involving the China Earthquake Authority's Department of Earthquake Science, the Chinese Academy of Sciences' Laboratory of Nonlinear Mechanics, and Peking University are underway to study the physics of catastrophic failure in rocks and link these to seismic observations.

As a follow up to the ACES cooperation and to take best advantage of complementary national programs and infrastructure, ACES participants have agreed to establish a research frontier institute for solid earth system simulation called the international Solid Earth Research Virtual Observa- tory, or iSERVO (www.iservo.edu.au). The institute will extensively use the Web, computational Grid technologies, and multitiered information architectures to let researchers manipulate simulation models and data by symbolic means in a way not previously possible. It will also involve developing a national node in each participating country linked via Web-based services and portals to enable different numerical simulation models, simulation software, geological models, and data constraints contributed by Australia, Japan, and the US. The models and data sets accessible within iSERVO will include several "standard" crustal fault system models (such as strike-slip, intraplate, and subduction).

The iSERVO Grid is being constructed from Web services to be consistent with Grid Forum standards. The system will use distributed computing including high-performance computers and distributed heterogeneous databases. These will be accessible through portals exploiting the new portlet standards. The institute nodes in each participating country will have research, software, and technical staff to drive continued development of the institute's virtual laboratory infrastructure, conduct high-impact simulation-based research of national importance using the virtual laboratory, and interface with the broader user community including the scientific community affiliated with the institute, external research users, government agencies, and industry users. dynamics of crustal fault systems, and geological processes such as fault formation, basin evolution, mountain building, and global geodynamics. The simulator system also has a high-level scripting language that insulates researchers from the parallel software layer and lets researchers easily change models without becoming experts in parallel computing. Moreover, it includes a cellular automaton module to let researchers study the Earth's complex system behavior.

\section{Initial ACES Work}

Starting in 1994, one of the authors (Peter Mora) started to develop the Queensland University Advanced Centre for Earthquake Studies (Quakes), a university research center, and an infrastructure in Australia for simulating the physics of earthquakes. The initial effort focused on developing a new particle-based simulation model termed the Lattice Solid Model (LSM) that lets researchers study earthquake nucleation and fault-zone processes. ${ }^{3-7}$ The model simulated fracturing behavior, frictional processes of rough fault surfaces, and fault gouge zones ${ }^{5}$ as well as frictional heating and heat transfer, thermomechanical coupling effects, and the effect of pore fluid migration resulting from pressure gradients induced by the frictional heating of pore fluids. ${ }^{3,6}$

Recognizing the magnitude of simulating all the Earth's processes led the center's developers to seek collaboration with complementary national and international efforts. This culminated in establishing the ACES international cooperation to link international efforts and the founding of the ACcESS national research facility. The simulation programs in Australia that merged to form ACcESS included

- particle-scale simulation,

- crustal fault system and seismic wave simulation,

- geological processes and geodynamics simulation,

- surface processes simulation,

- tectonic reconstruction, and

- visualization and parallel software.

These Australian solid earth simulation efforts involve the University of Queensland, Monash University, the Victorian Partnership for Advanced Computing, Australian National University, Melbourne University, RMIT University, and the University of Western Australia. 


\section{ACcESS Development}

ACcESS (see www.access.edu.au) is one of the two new national facilities in the physical sciences that were established under the Commonwealth government's Major National Research Facilities (MNRF) 2003-2007 program. Headquartered at the Earth Systems Science Computational Centre (www.esscc.uq.edu.au) — formerly at Quakes—at the University of Queensland, the AUS\$15 million facility is being funded by the Australian Commonwealth government, the Queensland government, the Victorian government, a consortium of the participant universities, two governmental agencies (the Commonwealth Scientific and Industrial Research Organisation and Queensland Department of Main Roads), and a leading parallel supercomputer vendor (SGI).

The ACcESS simulator consists of software systems and numerical models for multiscale, multiphysics simulations of solid earth systems and thematic supercomputer hardware. The simulator provides the means to simulate the dynamics of the entire Earth, including processes ranging from mantle and crustal evolution, to crustal dynamics and mineralization, to granular and fault-zone dynamics.

The simulator parallel software system includes particle simulation modules, a continuum solver library (Finley) based on finite elements, and various other modules, including a second continuum solver package based on the particle-in-cell and finite element methods (Snark) and a platetectonics reconstruction package (PlatyPlus). Users control the simulator system through GUIs and a high-level scripting interface (Escript) that lets them easily specify and map the partial differential equations (PDEs) and particle simulations onto the parallel software system. A cellular automaton and complex system module (ESyS-CA) lets researchers readily conduct phase-space studies and summarize results on an automatically generated Web page.

The ACcESS software enables simulation at scales from the microscale (Figure 1), to the crustal scale (Figure 2), ${ }^{8}$ up to the mantle and global scales (Figure 3).

The ACcESS facility's goal is to provide predictive modeling capabilities to Australian researchers and international collaborators that drive scientific advancement and breakthroughs in solid earth systems science and technology. Examples include quantum leaps in understanding the Earth's evolution at global, crustal, regional, and microscopic scales; predicting geological processes such as tectonics and mineralization; understanding the rock failure leading to mining technology innovations; new understanding

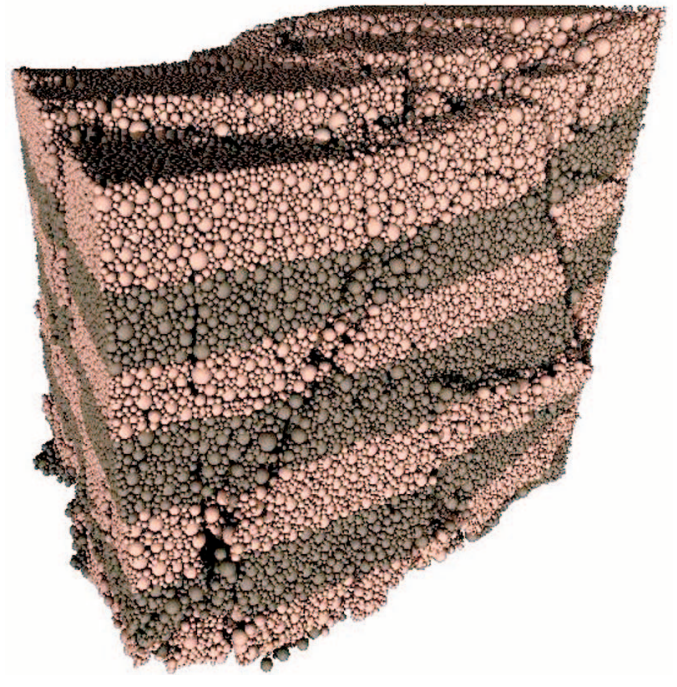

Figure 1. Image showing a 3D-particle-based simulation of the fracture and collapse of a rock structure. The rock was represented as an assemblage of random-sized grains cemented together by elasticbrittle bonds with horizontal bands of color added at the start of the simulation to let researchers easily visualize the fracturing. The simulation was generated by the ACCESS LSM software module.

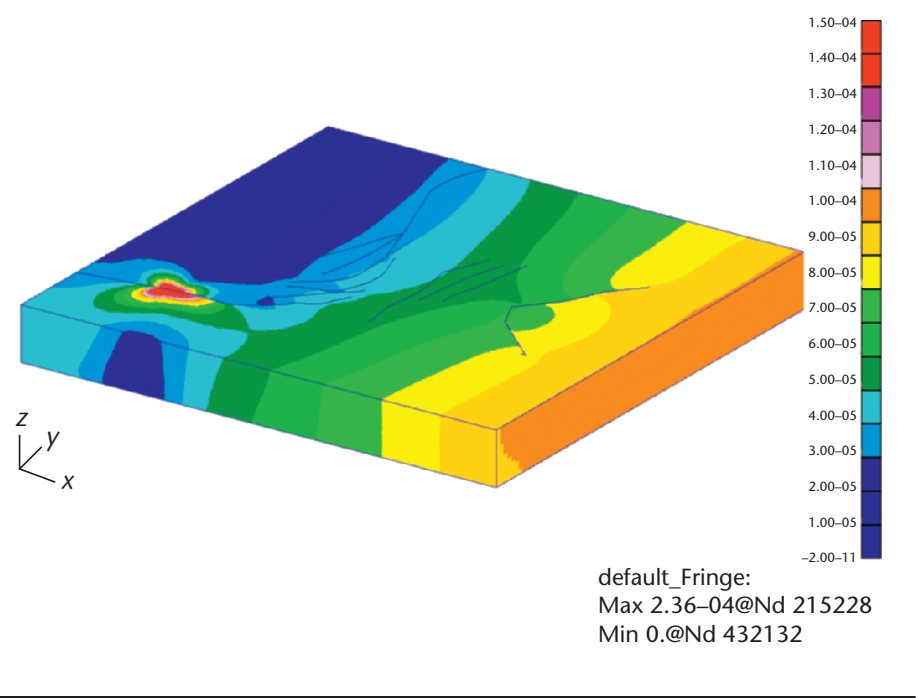

Figure 2. Image from a 3D simulation of the South Australian fault system. ${ }^{8}$ The different colors represent velocity.

of hot dry rock geothermal reservoir systems; and new knowledge of the physics of the crustal fault systems required to forecast earthquakes.

In 2003, SGI and the Earth Systems Science Computational Centre installed the ACcESS supercomputer hardware at the University of Queensland. It consists of a 208-processor SGI Altix 3700 parallel supercomputer with a peak per- 


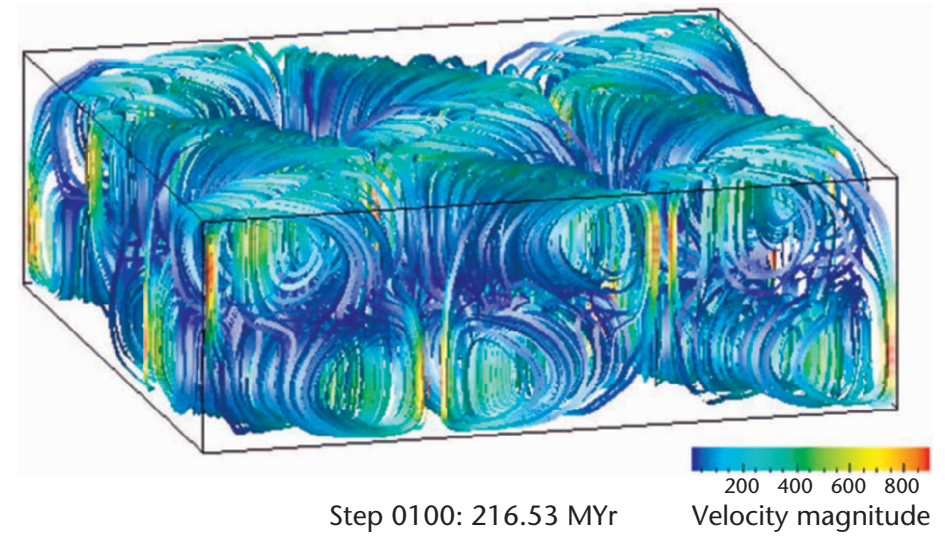

Figure 3. Image from a 3D mantle-convection simulation showing velocity streamlines. ${ }^{9,10}$ Colors represent the magnitude of local velocity (see legend); the Rayleigh number is $R a=106$. The normal velocities are fixed on the boundaries and the temperatures on the top and bottom of the model are prescribed.

formance of 1.1 Tflops $\left(1.1 \times 10^{9}\right.$ floating-point [numerical] operations per second), 5 Tbytes of disc, and 208 Gbytes of memory. The Queensland government's Smart State Research Facilities Fund funded the supercomputer, and it is available for solid earth simulation research by Australian scientists and international collaborators.

\section{Escript: A High-Level \\ Computational Framework}

A correct model makes computer simulation successful, but getting the model right is not easyparticularly in geosciences. We can quickly add an extra term into a differential equation or introduce a new equation or effect into a numerical model on paper. However, getting this type of change into a computer program can be a hard and timeconsuming exercise, despite the extensive list of available numerical libraries and environments. Most earth scientists and solid earth system modelers are more focused on scientific questions relating to the solid earth and aren't highly skilled at software development. This has led many researchers to either search for analytical or numerical solutions for very simple cases-not really what we want to do-or rely on data analysis to motivate cartoon-like pictures to describe the earth system's physics and evolution. Alternatively, researchers use whatever existing simulation capability is easy for them to access, even if the numerical model might have undesired limitations for the research question being posed.

How do we overcome this? The quick answer is to teach earth scientists and modelers how to pro- gram, but it's worth looking a bit deeper into the way we implement numerical simulations. In fact, a tight link exists between the numerical methods and data representation with the actual model. A typical example is classical finite element codes, which keep connectivity information and physical properties in the same data structure for each individual element. For an experienced programmer, the advantages might be obvious - in particular, when working with parallelized codes-but modifying the model requires modifying the source code, which necessitates a good knowledge of the program code.

An extra software layer is necessary to crack the link between the model and the numerics. This is the layer where all the model formulation-all the mathematics-happens. As part of developing the ACcESS facility, we're creating a programming environment called Escript to implement this idea. ${ }^{9-11}$ Initially, the Escript language will provide researchers with high-level access to models and parallel software based on PDEs, which are the core of most relevant models in earth sciences. The language will incorporate a prototype particle scripting language used to drive the lattice solid particle simulation model and let researchers simulate hybrid particle-continuum systems.

We can describe the functionality of Escript's mathematics layer with a simple example: the timedependent temperature diffusion that is essentially controlled by density, permeability, and the heat source. The model is a differential equation containing spatial and time derivatives. By applying a time-integration scheme, we end up with a linear spatial differential equation to be solved at each time step. The coefficients are defined by expressions of the model input parameters' density and permeability, the heat source, and the last time step's temperature. As far as the model description is concerned, we can solve the problem without describing the representation of temperature on the computer or how we discretize the differential equations. These aspects of the simulation are hidden from researchers so they can focus on the model and the problem's basic physics rather than computer science and software issues. The differential equation solvers plugged into Escript determine these issues without involving the user.

Escript is an extension of the Python objectoriented language, which is easy to use even for people with no programming background. In addition, Python is lenient regarding class definitions, so we can change the object types used to define coefficients of differential equations without code modifications. Researchers can change model pa- 
rameters such as the heat source in the diffusion script from a Python floating-point number to a more general Escript data object. The values, which are the result of another differential equation, can be read from an external data file. Behind the scenes, Escript resolves the hand-over of the coefficient to the differential equation solver, regardless of the representation chosen when the coefficient was created.

By hiding the parallel software and data representation issues from users, Escript lets us develop highly portable simulation codes; this portability not only refers to the computer platform, but also to the discretization techniques and implementations being used to solve the differential equations. We've successfully applied scripts to mantelconvection simulation using both a finite element discretization and a finite difference discretization scheme and both the OpenMP and messagepassing interface (MPI) parallel environments. Currently, an extension of the concept of the abstraction from the numerical layer is implemented for software components doing time-series analysis and visualization.

Escript ensures a high level of abstraction from numerical software components, letting researchers apply Grid technology to simulations. We can translate the scripts into workflow distributed across the Grid. The Escript programming environment is an important cornerstone toward developing data Grids in efficient and easy-to-use simulations.

As a pilot project for iSERVO, Australia and the US collaborated to develop a prototype Web portal using Escript to drive simulation codes from the two countries. This collaboration involved the ACcESS MNRF and the University of Queensland's Earth Systems Science Computational Centre, the Community Grid Labs at Indiana University, and NASA's Jet Propulsion Laboratory. In this work, researchers at Indiana's Community Grid Labs and the University of Queensland's Earth Systems Science Computational Centre developed a prototype Web portal in which a window for Escript was used to define a problem that interfaced to either a US finite element code developed by JPL or an Australian finite element code developed by ACcESS and Earth Systems Science Computational Centre to run the problem on a supercomputer at a specified location. Researchers then visualized the results of the simulation in another window. This kind of Web-based simulation environment using scripting to define problems and parallel simulation software contributed by different groups minimizes duplication of effort and

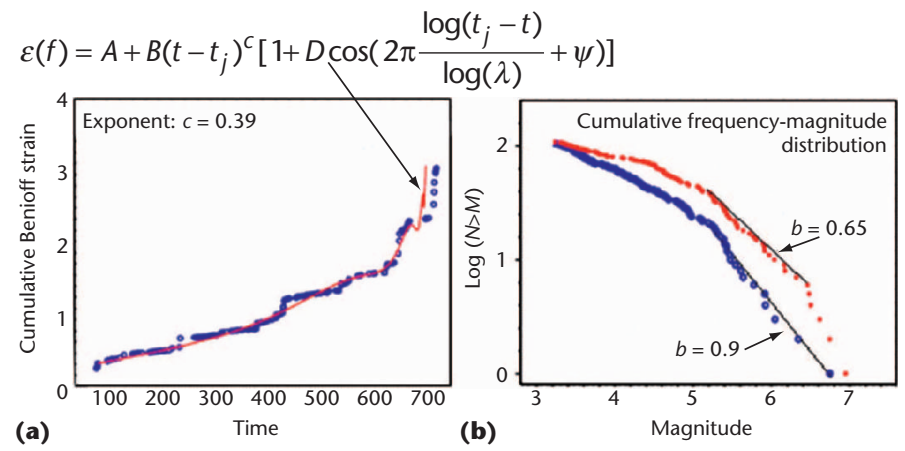

Figure 4. LSM simulation results exhibiting features consistent with the Critical-Point Hypothesis for earthquakes. (a) Cumulative Benioff strain in a lattice solid elastodynamic simulation of a granular region subjected to a constant normal stress and sheared at a constant rate. ${ }^{6}$ (b) Cumulative number of events larger than a given size for the first (blue) and last half (red) of the simulated earthquake sequence in (a), excluding the large events terminating the sequence. The distributions are power laws (linear on the log-log plot), at least for moderate to larger event sizes. The rollover for the smaller event sizes is believed to be a finite model size effect.
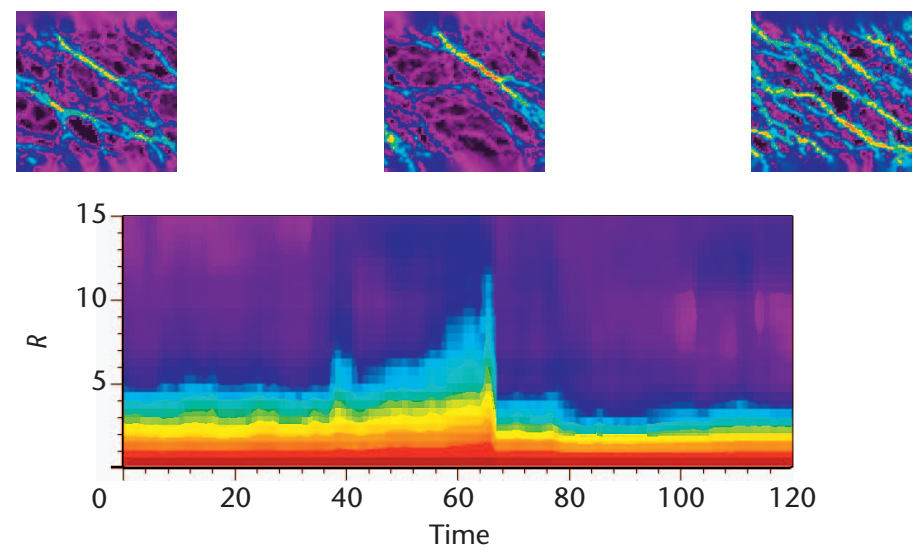

Figure 5. Stress field in a granular model representing a fault zone or system (top) and stress correlation function (bottom). The lower plot shows a growth in correlation lengths in the lead up to the catastrophic failure at model time 66 and a sharp drop in correlation lengths when the large event occurred. Note that the hot and cold colors depict high and low values, respectively, and time equals 66 here is equivalent to time equals 700 in Figure 4a).

makes simulation more accessible and effective as a research tool for the community.

\section{Microscale Simulation of Fault} Mechanics and Earthquake Physics

The $\mathrm{LSM}^{3,4,6,7}$ is a particle-based model for simulating discontinuous systems such as heterogeneous rock, fault zones, fault gouge, and granular systems. 

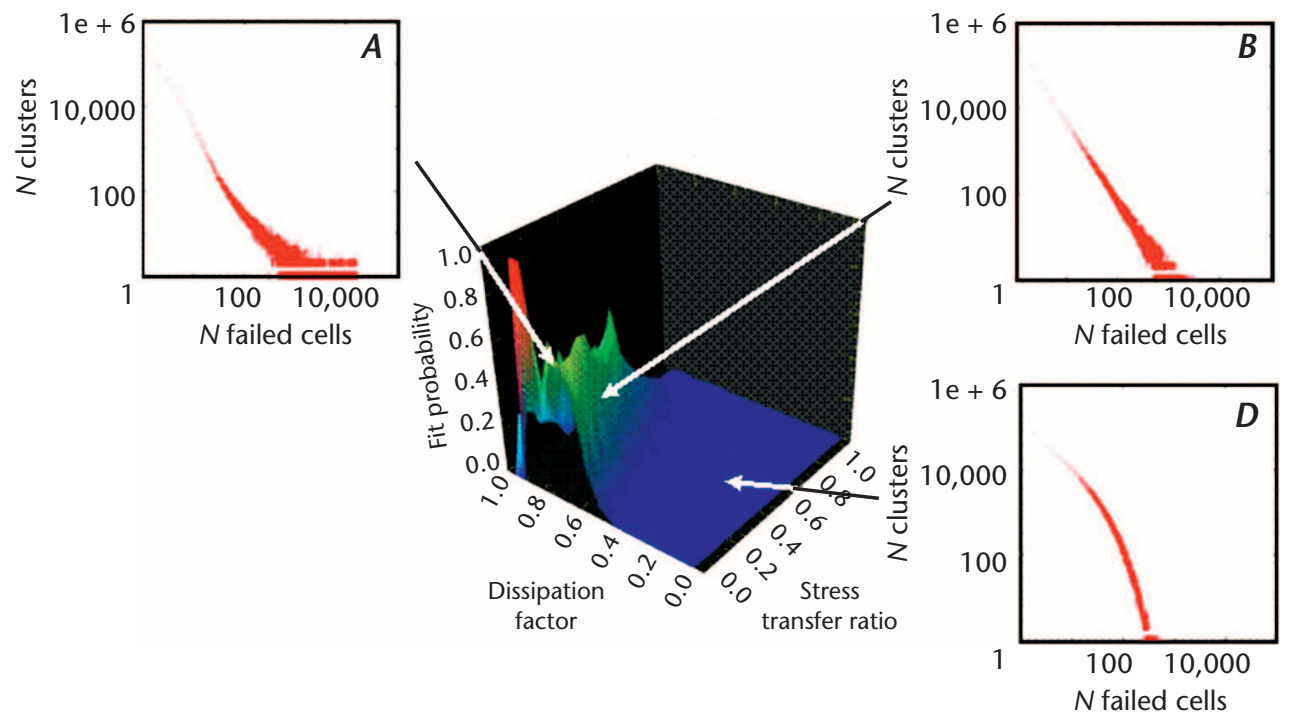

Figure 6. Phase-space plot. The fit probability of a power-law accelerating energy release prior to large events for short-range cellular automaton (CA) models is a function of the dissipation factor and stress transfer ratio. Two regimes are identified as being $A$ (forecastable equals high fit probability) and $D$ (not forecastable equals low fit probability). A transitional regime (borderline forecastable equals moderate fit probability) separates the two. The frequency-magnitude statistics indicate overabundance of large events in regime $A$, power-law statistics in regime $B$ (the transitional regime), and a deficit of large events in regime $D$.

Researchers have used it to simulate the nonlinear dynamics of earthquakes, ${ }^{4,6,12}$ localization phenomena in fault zones, ${ }^{5}$ heat flow around faults, ${ }^{5}$ thermomechanical and thermoporous coupling effects, ${ }^{3,6}$ the micromechanics of fault gouge layers, ${ }^{13}$ and earthquake forecasting. ${ }^{6,14-16}$ A parallel software system implementing this model is being developed by ACcESS staff within the ACcESS software system and will be accessible to researchers through the Escript scripting system and GUIs, with a beta software release scheduled in 2006. Recent research using the LSM has shown evidence for an underlying physical mechanism for earthquake forecasting compatible with the CriticalPoint Hypothesis for Earthquakes ${ }^{16}$ - namely, the hypothesis that the Earth's crust is not perpetually in a critical state such that a large earthquake may occur (see Figures 4 and 5). Instead, the Earth's crust undergoes cycles in which smaller earthquakes build up stress correlations and cause the crust to approach a critical state when a large event can occur. When this happens, the crust is perturbed away from criticality and the cycle repeats.

Figure 4 shows that lattice solid particle models of granular layers subjected to shearing frequently exhibit accelerating energy release and an increased rate of moderate to large earthquakes prior to large simulated earthquakes. Also, analysis of the stress evolution in the granular region indicates that the correlation length of the deviatoric stress grows in the lead up to the large events that terminate the sequence shown in Figure 5. These results are consistent with the Critical-Point Hypothesis for Earthquakes and provide simulation-based evidence for a physical mechanism for forecasting catastrophic failure of discontinuous elastodynamic systems and, hence, for forecasting earthquakes.

The particle simulations are computationally expensive, so it's useful to compare the LSM results to those obtained via the cellular automaton (CA) models that represent simplified analogs for earthquake physics. These CA models let us conduct many simulations with different parameters representing variables such as frictional losses. This kind of parameter space study is called a phase-space study and is used to explore the nature of the nonlinear dynamics of the simplified fault system expressed in the CA model. We can then compare the CA results to the more sophisticated LSM results to gain insights into the nature of the more realistic model's nonlinear dynamics and thus the real Earth. A CA simulation module is being developed by ACcESS staff within the ACcESS software system that includes the ability to submit many simulations to study phase space. A beta software release will be available in 2006 . 
CA models representing simplified analogs for earthquake physics also exhibit the accelerated energy release and growth of correlation lengths prior to large events. ${ }^{17,18}$ Phase-space studies have revealed two regimes of phase space (see Figure 6). Simulations within the first regime exhibit an overabundance of large events relative to power-law event statistics and accelerating energy release prior to large events- that is, the larger simulated earthquakes are forecastable. Simulations within the second regime exhibit a deficit of large events relative to power-law event statistics and no accelerating energy prior to large events-that is, the larger simulated earthquakes aren't forecastable. Powerlaw or Gutenburg-Richter event statistics are only seen near the transition between the forecastable and unforecastable regimes of phase space.

Recent results comparing CA and LSM models ${ }^{6,16-18}$ suggest that discontinuous elastodynamic systems - and thus crustal fault systems-lie close to the borderline separating a region of phase space in which we might forecast earthquakes and another region of phase space in which we can't forecast them (see Figure 6). This might explain often contradictory observational evidence for earthquake forecastability. If the Earth's crust lies near the transitional regime, slight variations in crustal parameters might determine whether earthquakes are forecastable in any given region. Hence, it's crucial to understand how the parameters of real crustal fault systems-viscosity, fault friction, loading rate, fault density, fault geometry, and so on-affect the system dynamics to provide a sound scientific underpinning for earthquake forecasting.

\section{Macroscale Simulation of Crustal Fault Systems}

The particle and CA simulation results we've described so far play a vital role in improving the understanding of fault zones' micromechanics and have shown evidence for an underlying physical mechanism for earthquake forecasting. However, we still might question whether the particle simulation results involving a granular layer are applicable to real crustal fault systems. The particle simulations model a discontinuous or granular system's elastodynamics. When grains suddenly slide past one another, elastic strain energy is released and converted to radiated seismic waves in the model. Such rupture events represent simulated earthquakes in the model, but a key difference between such ruptures and the ruptures in an earthquake fault system is that the model's internal geometry changes as grains move. However, in real fault systems, there is no sig-

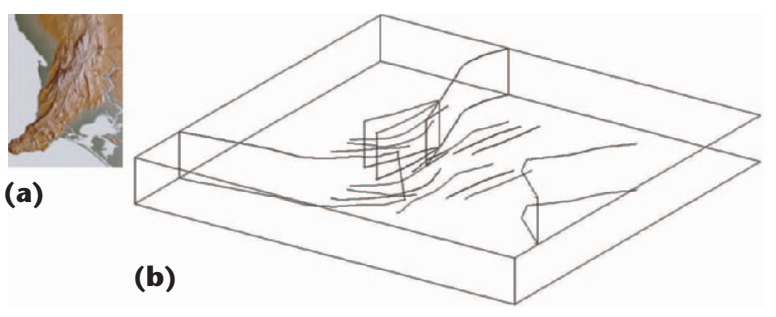

Figure 7. The South Australian fault system model. (a) Image of the region of South Australia being discretized and (b) 3D fault model of the South Australian fault system.

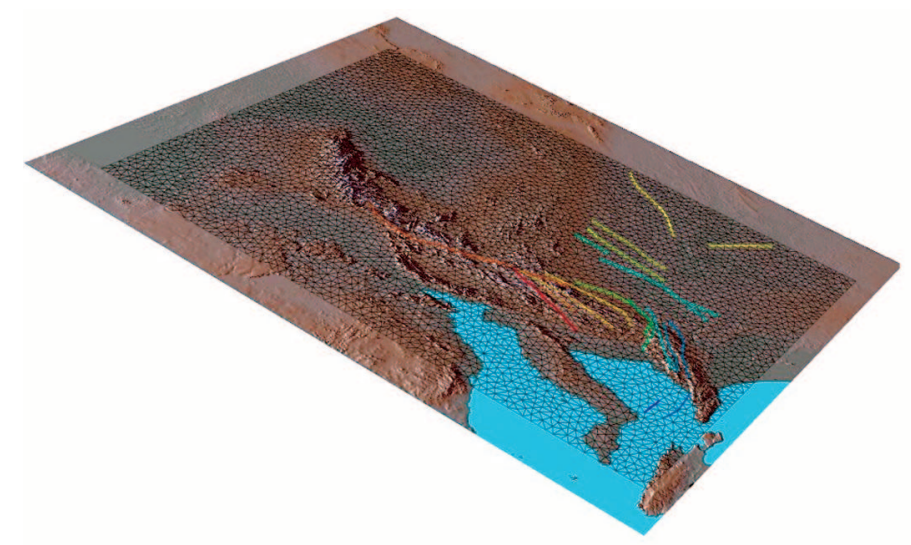

Figure 8. A finite element discretization of the South Australian fault system. The solid lines depict the random triangular mesh used to discretize the region of South Australia being modeled.

nificant geometric change to the fault system as a result of a single slip event. For this reason, an ability to simulate crustal fault systems more realistically is vital for studies of the physics of earthquakes and interacting fault systems.

To solve this problem, we're developing simulation capabilities to model the dynamics of crustal fault systems based on the finite element method. ${ }^{19}$ In this approach, a crustal fault system is discretized onto a finite element mesh with faults represented as discontinuities between volumes of material. Figures 7 and 8 show an example of such a discretization for the South Australian fault system, ${ }^{8}$ and Figure 9 shows a snapshot of the equivalent stress-rate distribution in a simulation. In these simulations, and others modeling the San Andreas California fault system, ${ }^{20}$ we've seen evidence for clustering earthquake activity and stress transfer effects on earthquake activity. This supports the need to model the system dynamics as a whole to better study the earthquake forecasting question.

We're currently integrating this capability into 


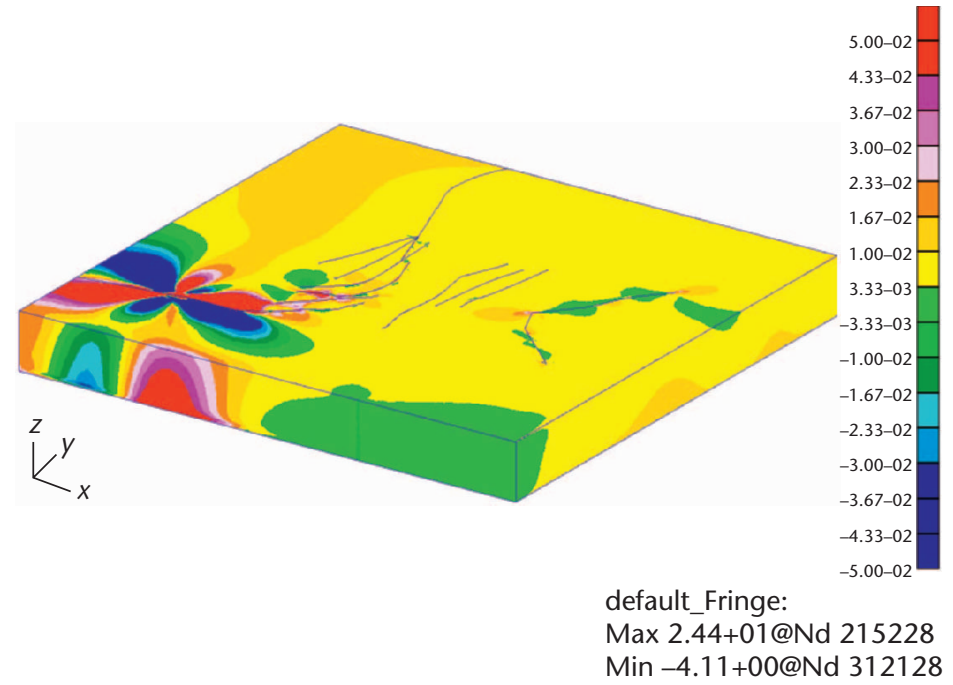

Figure 9. A snapshot from a simulation of the South Australian fault system showing the equivalent stress-rate distribution. Hot colors represent positive values of equivalent stress rate (increasing stress), and cold colors represent negative values of equivalent stress rate (decreasing stress).

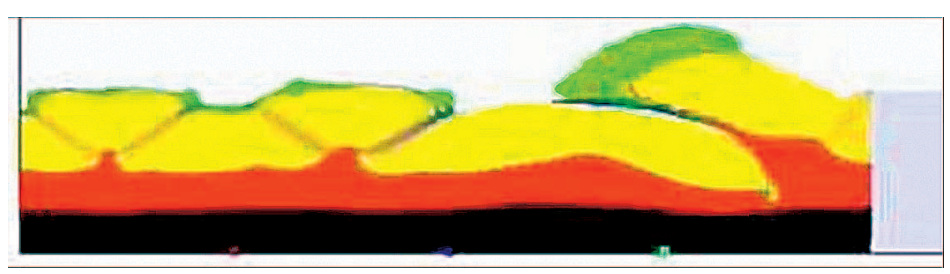

Figure 10. Snapshot of a simulation using the particle-in-cell method to model formation of thrust faults. A system consisting of a viscoplastic upper layer; a nonlinear, ductile middle layer; and a highly viscous lower layer was initially extended. Sediments were deposited in the faultbounded basins, which developed spontaneously. On overall system compression, the basin-bounding faults were reactivated as thrusts.

the high-level Escript computational environment and developing visualization and analysis tools to probe the earthquake question. In our approach, we'll model stress buildup using a quasistatic approach until an earthquake nucleates in the model, at which time we'll switch over to a fully dynamic computational approach to simulate dynamic rupture and the associated stress redistribution. The analysis tools will provide earthquake hypocenters and magnitudes, as well as stress correlation functions, to let researchers study the physics of interacting fault systems and compare the simulated observables to field observations. Presently, a 2D prototype module has been developed within the Escript framework in which the scripting language specifies the PDEs. A fully $3 \mathrm{D}$ prototype module has also been developed that interfaces to the Escript framework but in which the PDEs themselves are not specified by the scripting language. These capabilities will be available in the 2006 beta software release.

Our simulation framework will ultimately be set up to enable simulations of the elastodynamics of interacting fault systems, including heat transfer, thermal coupling effects, viscoelastic effects, and pore fluid flow induced by stress fluctuations. These capabilities-which we anticipate will be available in 2007-will provide a powerful tool to study complex crustal system behavior such as the physics of interacting fault systems and geothermal reservoirs. This tool provides a means to achieve breakthroughs in understanding leading to an improved ability to forecast earthquakes, improved understanding of crustal evolution and mineralization processes, and exploitation of new green energy resources such as hot fractured rock geothermal energy.

\section{Simulating Geological Processes and Mantle Convection}

Studying long-time-scale geological processes such as mountain building, fault formation, and basin evolution requires a different kind of computational machinery that can simulate large deformations. For this purpose, we're developing computational models based on the particle-in-cell method. ${ }^{21,22}$ Figures 10 and 11 show example 2D simulations to illustrate this method's capability. ${ }^{23,24}$

Figure 10 depicts a snapshot of layers that were first extended and then compressed, leading to the formation of thrust faults; Figure 11 shows a snapshot of a salt dome in which a lighter salt lens pushes upward and deforms overlying layers, resulting in fault displacements. Such simulations help bridge the gap between long-lived mantle processes and the time scale of fault interactions in the brittle crust. One of the most fundamental questions in geodynamics is the manner in which strains derived from relative plate motions are manifested in the brittle upper part of the crust. We can examine the partitioning of the strain between the continuum deformation between faults and slip on the faults themselves in the context of the rheological layering in the lithosphere as well as the feedback between surface deformation patterns and the response of deep mantle flow. Related to this topic is the question of the relative deformation of crust and mantle-when do we expect to see delamination and the spontaneous development of 3D structure, and what is the role of lateral strength variations in 
the crust/mantle lithosphere in producing $3 \mathrm{D}$ structure from a 2D driving force? ? $^{24}$

Deep earth processes such as mantle convection are at the heart of understanding how the Earth works, including the evolution of its topography. However, these processes remain at best poorly understood because of the substantial technical challenges associated with modeling mantle convection, plate tectonics, and the topography's associated evolution. Mantle convection is characterized by strongly variable (that is, stress-, temperature-, and pressure-dependent) viscosities. The lithosphere exhibits the critical processes such as fracture and shear-zone deformation (strain localization) that are physically distinct from the viscous flow deeper in the mantle, and they occur on fundamentally different (smaller) length scales. Researchers have barely touched the feedback of free surface effects and the influence of topography. In addition, the mantle is chemically heterogeneous, is replete with silicate melts and volatiles, and has numerous pressure- and temperature-induced structural (phase) changes that affect its dynamics.

ACcESS is developing a suite of software that is uniquely suited to tackle the computational simulation of the interplay of free surface effects (topography), plate tectonics, and deep earth processes such as mantle convection and magmatism. ${ }^{23,25}$ Modeling of mantle convection also provides a means to study mineralization processes. ${ }^{26}$ However, from the mathematical nature of the underlying models, the response of the simulations will in many cases depend on the often poorly constrained initial conditions. Hence, the mantle convection models must be more closely integrated into the real world through direct data assimilation and directly tested against various observations.

Global-scale simulation of mantle convection is necessary for studying Earth's evolution. Figure 12 shows a simulation using the Terra software. ${ }^{27-30}$ We're integrating this simulation capability into the ACcESS computational system to make global-scale simulation more accessible to researchers. Ultimately, researchers can use tectonic reconstructions (see Figure 13) as the surface motion constraints for the global models. This kind of integration of geological observations to constrain past surface displacements is important because these constraints influence internal flow patterns. The global-simulation capability provides a powerful means to answer fundamental questions about the Earth's evolution and dynamics and to gain insights into plume and mantle dynamics. This has both scientific significance and economic implications in terms of exploring for massive new mineralization deposits.

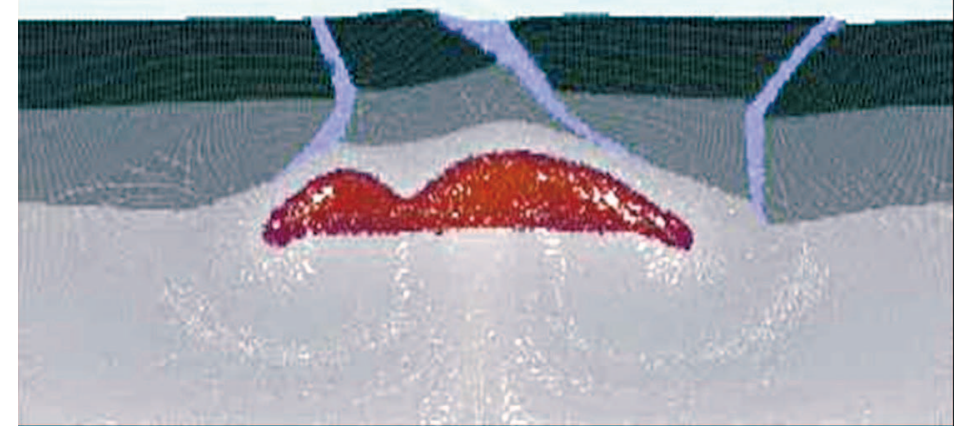

Figure 11. Snapshot of a simulation using the particle-in-cell method to model the formation of a salt dome and resultant deformation and faulting. In the simulation, a low-density lens representing a buried salt layer underneath highly viscous rock layers with prescribed weak zones (representing faults) shown in blue is pushed up into the overlying layers, deforming the layers and displacing the faults.

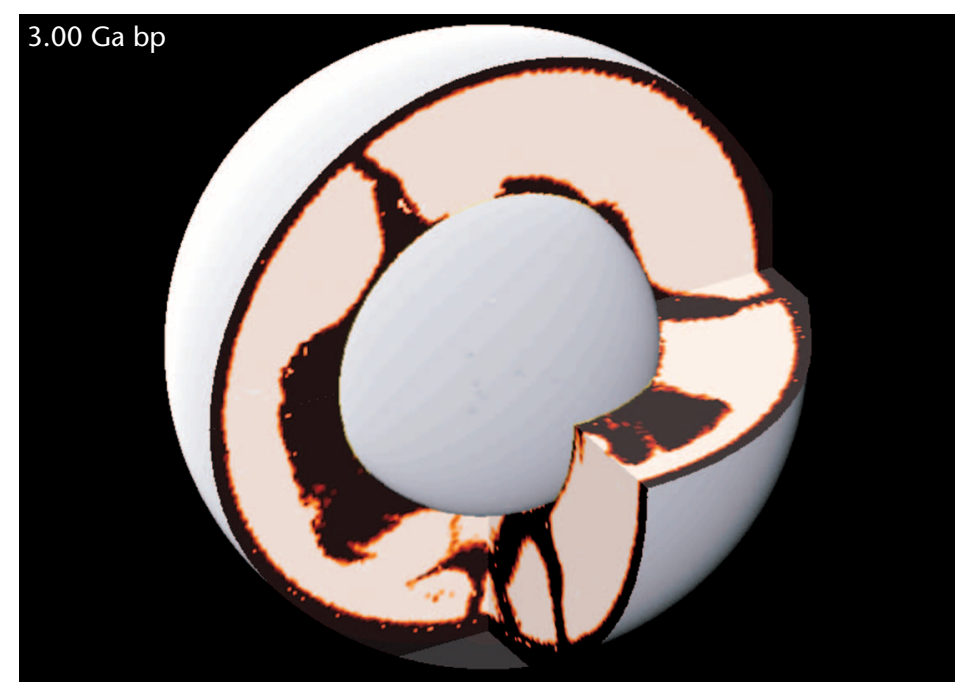

Figure 12. Snapshot after 1.49 gigayears (Gyr) of $4.49 \mathrm{Gyr}$ of a mantleconvection simulation using the Terra software. ${ }^{30}$ Pristine material is white, and dark colors represent material that has been altered near the surface-for example, by partial melting or degassing. The aim of this kind of model is to reconcile geophysical and geochemical constraints on the evolution of Earth's mantle.

olid earth systems simulation is now becoming feasible from the microscopic to the global scale. The ACES international cooperation has shown development of simulation capabilities for solid earth phenomena that are beyond the ability of a single group or country. Each country has different strengths, computational approaches, and laboratory and field observational systems. This range of numerical models is required to 


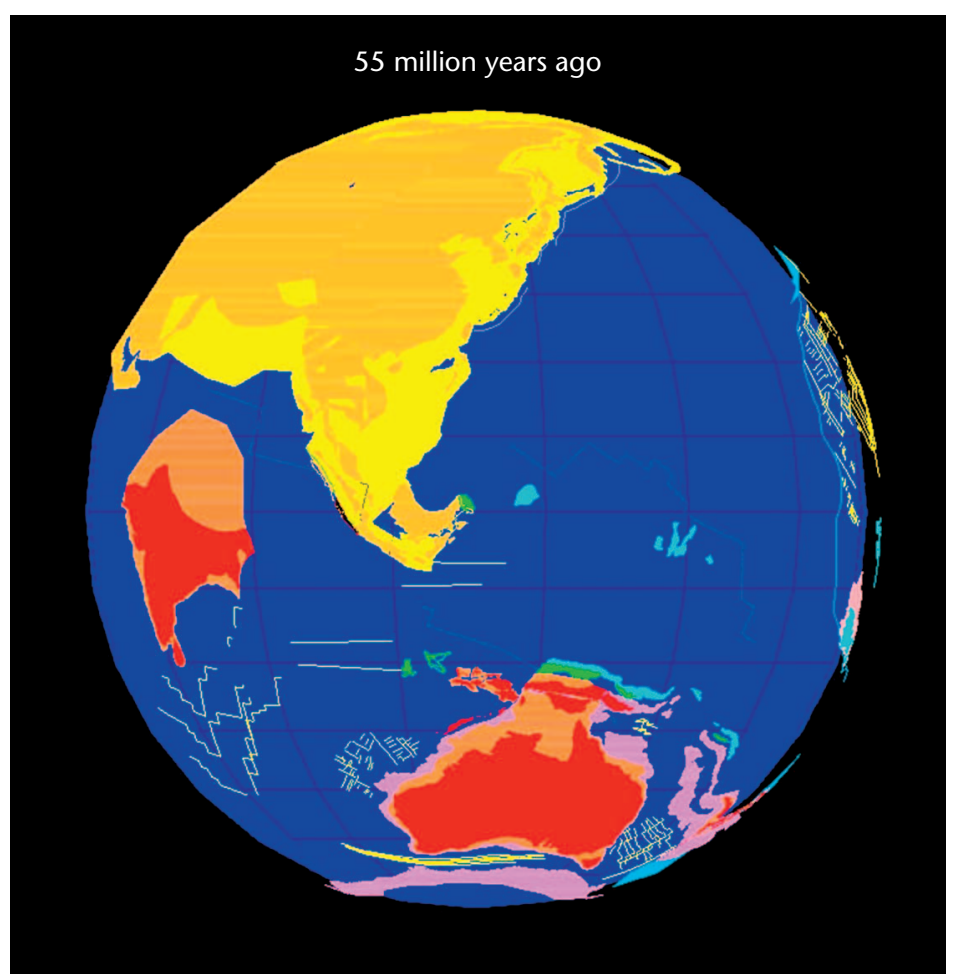

Figure 13. Snapshot from a plate-tectonic reconstruction using the PlatyPlus software developed by Gordon Lister of the Australian National University and his group at Monash University. Such geological-observation-based surface movements can help researchers constrain surface-boundary conditions in global mantleconvection simulations.

model the entire earth system, and calibration is required to ensure that results obtained using these models match with the different laboratory and field observations available in each country. For this reason, international groups have agreed to work toward establishing iSERVO to build the ACES cooperation. iSERVO aims to collaboratively develop a computational infrastructure-accessible through Web portals - that combines models developed across the international community and to conduct collaborative research to solve problems of global significance such as earthquake forecasting, green energy development, and environmental management.

\section{Acknowledgments}

We gratefully acknowledge support from the Australian Computational Earth Systems Simulator national research facility, the Commonwealth of Australia MNRF program, the Australian Research Council, the Queensland State Government, the University of Queensland, and SGI. The Queensland State
Government Smart State Research Facility Fund and SGI funded the ACCESS supercomputer.

\section{References}

1. A. Donnellan et al., Computational Earthquake Science, Part I, Birkhäuser, 2004.

2. A. Donnellan et al., Computational Earthquake Science, Part II, Birkhäuser, 2004.

3. S. Abe, P. Mora, and D. Place, "Extension of the Lattice Solid Model to Incorporate Temperature Related Effects," Pure and Applied Geophysics, vol. 157, nos. 11 and 12, 2000, pp. 1867-1887.

4. P. Mora and D. Place, "Simulation of the Frictional Stick-Slip Instability," Pure and Applied Geophysics, vol. 143, nos. 1-3, 1994, pp. 61-87.

5. P. Mora and D. Place, "The Weakness of Earthquake Faults," Geophysical Research Letters, vol. 26, no. 1, 1999, pp. 123-126.

6. P. Mora et al., "Lattice Solid Simulation of the Physics of Earthquakes: The Model, Results and Directions," GeoComplexity and the Physics of Earthquakes, J.B. Rundle, D.L. Turcotte, and W. Klein, eds., Am. Geophysical Union, 2000, pp. 105-125.

7. D. Place and P. Mora, "A Lattice Solid Model to Simulate the Physics of Rocks and Earthquakes: Incorporation of Friction," J. Computational Physics, vol. 150, no. 2, 1999, pp. 1-41.

8. H.L. Xing and P. Mora, "Construction of an Intraplate Fault System Model of South Australia and Simulation Tool for the iSERVO Institute Seed Project," submitted to Pure and Applied Geophysics, 2005; www.aces-workshop-2004.ac.cn/html/ext.htm.

9. M. Davies, L. Gross, and H. Mühlhaus, "Scripting High Performance Earth Systems Simulations on the SGI Altix 3700," Proc. 7th Int'l Conf. High-Performance and Grid Computing in the Asia Pacific Region, IEEE CS Press, 2004, pp. 244-251.

10. M. Davies, H.B. Mühlhaus, and L. Gross, "The Rapid Development of High Performance Numerical Models in Mantle Convection," Proc. 4th ACES Workshop, 2004; www.aces-workshop2004.ac.cn/html/ext.htm.

11. L. Gross, M. Davies, and J. Gerschwitz, "A High-Level Programming Language for Modelling the Earth," Proc. 4th ACES Workshop, 2004; www.aces-workshop-2004.ac.cn/html/ext.htm.

12. S. Abe, S. Latham, and P. Mora, "Simulation of the Dynamic Rupture of a Rough Planar Fault in 3D using the Lattice Solid Model," Proc. 4th ACES Workshop, 2004; www.aces-workshop2004.ac.cn/html/ext.htm.

13. S. Abe and K. Mair, "Grain Fracture in 3D Numerical Simulations of Granular Shear," Geophysical Research Letters, vol. 32, no. 5, article no. LO53052005.

14. P. Mora et al., "Simulation of the Load-Unload Response Ratio and Critical Sensitivity in the Lattice Solid Model," Pure and Applied Geophysics, vol. 159, no. 10, 2002, pp. 2525-2536.

15. Y.C. Wang et al., "Statistical Tests of Load-Unload Response Ratio (LURR) Signals Using the Lattice Solid Model (LSM): Implication to Tidal Triggering and Earthquake Prediction," Pure and Applied Geophysics, vol. 161, nos. 9 and 10, 2004, pp. 1829-1839.

16. P. Mora and D. Place, "Stress Correlation Function Evolution in lattice Solid Elasto-Dynamic Models of Shear and Fracture Zones and Earthquake Prediction," Pure and Applied Geophysics, vol. 159 , no. 10,2002 , pp. 2413-2427.

17. D. Weatherley, P. Mora, and M. Xia, "Long-Range Automaton Models of Earthquakes: Power Law Accelerations, Correlation Evolution and Mode Switching," Pure and Applied Geophysics, vol. 159, no. 10, 2002, pp. 2469-2490.

18. D. Weatherley and P. Mora, "Accelerating Precursory Activity within a Class of Earthquake Analogue Automata," Pure and Applied Geophysics, vol. 161, nos. 9 and 10, 2004, pp. 2005-2019.

19. H.L. Xing, P. Mora, and A. Makinouchi, "Finite Element Analysis 
of Fault Bend Influence on Stick-Slip Instability along an IntraPlate Fault," Pure and Applied Geophysics, vol. 161, nos. 9 and 10, 2004, pp. 2091-2102.

20. H.L. Xing and P. Mora, "Finite Element Modeling of Interacting Fault Systems," Proc. 4th ACES Workshop Abstracts, 2004, pp. 112-114; www.aces-workshop-2004.ac.cn/html/ext.htm.

21. L. Moresi, F. Dufour, and H.B. Mühlhaus, "A Lagrangian Integration Point Finite Element Method for Large Deformation Modelling of Viscoelastic Geomaterials," J. Computational Physics, vol. 184, no. 2, 2003, pp. 476-497.

22. D. Sulsky, Z. Chen, and H.L. Schreyer, "A Particle Method for History-Dependent Materials," Computer Methods in Applied Mechanics and Eng., vol. 118, nos. 1 and 2, 1994, pp. 179-196.

23. L. Moresi, F. Dufour, and H.B. Mühlhaus, "Mantle Convection Modelling with Viscoelastic/Brittle Lithosphere: Numerical Methodology and Plate Tectonic Modeling," Pure and Applied Geophysics, vol. 159, no. 10, 2002, pp. 2335-2356.

24. H.B. Mühlhaus et al., "Large Amplitude Folding in Finely Layered Viscoelastic Rock Structures," Pure and Applied Geophysics, vol. 159, no. 10, 2002, pp. 2311-2333.

25. H.B. Mühlhaus, L. Moresi, and M. Cada, "Emergent Anisotropy and Flow Alignment in Viscous Rock," Pure and Applied Geophysics, vol. 161, nos. 11 and 12, 2004, pp. 2451-2463.

26. C. Zhao et al., "Finite Element Modelling of Three-Dimensional Steady State Convection and Lead/Zinc Mineralisation in Fluid Saturated Rocks," J. Computational Methods Science Eng., vol. 3, no. 1, 2003, pp. 73-89.

27. J.R. Baumgardner, "Application of Supercomputers to 3-D Mantle Convection," The Physics of the Planets, S.K. Runcorn, ed., John Wiley \& Sons, 1988, pp. 199-231.

28. J.R. Baumgardner, "Three-Dimensional Treatment of Convective Flow in the Earth's Mantle," J. Statistical Physics, vol. 39, nos. 5 and 6, 1985, pp. 501-511.

29. H.-P. Bunge and J.R. Baumgardner, "Mantle Convection Modeling on Parallel Virtual Machines," Computers in Physics, vol. 9, no. 2, 1995, pp. 207-215.

30. K.-D.Gottschaldt, Vermischung in 3D sphärischen Konvektionsmodellen des Erdmantels [Mixing in 3D Spherical Models of the Earth Mantle], doctoral dissertation, Chemisch-Geowissenschaftliche Fakultät, Friedrich-Schiller-Universität, 2003.

Peter Mora is a professor at the Earth Systems Science Computational Centre at the University of Queensland, Australia. His technical interests include earthquake physics, computational earthquake science, and computational geophysics. Mora has a PhD in geophysics from Stanford University. He is a member of the American Geophysical Union, the American Association for the Advancement of Science, and the Society of Exploration Geophysicists. Contact him at director@ esscc.uq.edu.au.

Hans Mühlhaus is a professor at the Earth Systems Science Computational Centre at the University of Queensland, Australia, and an adjunct professor at the University of Western Australia. His technical interests include computational geodynamics, generalized continuum theories, and rheologies. Mühlhaus has a PhD in civil engineering from the University of Karlsruhe. He is a member of the Japanese Geotechnical Society for Soils and Foundations and the Australian Academy of
Science Theoretical and Applied Mechanics Group. Contact him at muhlhaus@esscc.uq.edu.au.

Lutz Gross is an associate professor at the Earth Systems Science Computational Centre at the University of Queensland, Australia. His technical interests include numerical methods for particle differential equations, scientific software, and high-performance computing. Gross has a PhD in mathematics from the University of Karlsruhe. He is a member of the Australian Mathematical Society.Contact him at I.gross@uq.edu.au.

Louis Moresi is an associate professor in the School of Mathematical Sciences at Monash University, Australia. His technical interests include planetary dynamics, plate tectonics, computational geophysics, and particle-based finite element methods. Moresi has a DPhil in geophysics from Oxford University. He is a member of the American Geophysical Union, the Royal Astronomical Society, and the Geological Society of Australia. Contact him at louis.moresi@sci.monash.edu.

Huilin Xing is a senior research fellow at the Earth Systems Science Computational Centre at the University of Queensland, Australia. His technical interests include computational mechanics and crustal dynamics, material science, and engineering. Xing has a PhD in material science and engineering. He is a member of the International Association of Computational Mechanics. Contact him at xing@esscc.uq.edu.au.

Dion Weatherley is a computational scientist at the Earth Systems Science Computational Centre at the University of Queensland, Australia. His technical interests include earthquake physics and computational geophysics. Weatherley has a PhD in geophysics from the University of Queensland. He is a member of the American Geophysical Union. Contact him at dion@ esscc.uq.edu.au.

Steffen Abe is a computational scientist at the Australian Computational Earth Systems Simulator. His technical interests include computational geophysics, discrete-element modeling, fault micromechanics, and earthquake rupture processes. Abe has a PhD in geophysics from the University of Queensland. Contact him at steffen@esscc.uq.edu.au.

Shane Latham is a computational scientist at the Australian Computational Earth Systems Simulator. His technical interests include parallel computation, scientific software development, and discrete-element modeling. Latham has a PhD in mathematics from the Australian National University. Contact him at slatham@access.edu.au. 